\title{
A New Mode Selection and Resource Reuse Strategy for V2X in Future Cellular Networks
}

\author{
Haider Daami R. Albonda, J. Pérez-Romero \\ Dept. Signal Theory and Communications \\ Universitat Politècnica de Catalunya (UPC) \\ Barcelona, Spain \\ [ haider.albonda, jorperez ] @tsc.upc.edu
}

\begin{abstract}
Recently, vehicle-to-vehicle (V2V) communications for future cellular systems have attracted considerable attention due to their potential benefits such as improved system capacity, spectral efficiency or delay reduction, and they enable new services related to intelligent transportation systems. V2V communications can be performed using two communication modes, namely cellular mode, based on uplink/downlink communications, and sidelink mode, which enables vehicles to communicate directly with other vehicles. However, selecting the appropriate operating mode according to the requirements of $\mathrm{V} 2 \mathrm{~V}$ services and allocating the radio resources to $\mathrm{V} 2 \mathrm{~V}$ communications becomes a challenging issue. In this respect, we propose a novel mode selection and resource reuse strategy to select the appropriate mode of operation and decide the amount of resource blocks (RBs) for $\mathrm{V} 2 \mathrm{~V}$ in cellular and sidelink modes, where multiple V2V links may share the same radio resources. A simulation-based analysis is presented to assess the performance of the proposed solution. The simulation results have shown that the proposed strategy improves network performance in terms of achievable throughput, outage probability, latency, and resource utilization.
\end{abstract}

Keywords-Cellular networks, Sidelink, LTE-V2X, Vehicle-toVehicle (V2V), Mode selection.

\section{INTRODUCTION}

Vehicle-to-Vehicle (V2V) communication has recently attracted great interest in the wireless communications community. In this context, direct V2V communications refer to a radio technology that allows users in close proximity to establish a direct link, bypassing the base station and the network infrastructure. Many important operational benefits can be achieved through the use of direct V2V connectivity, particularly in network load balancing to avoid deterioration in the quality of service (QoS) as well as to achieve significant system improvements in terms of network capacity, data rate and power consumption [1].

Various solutions for $\mathrm{V} 2 \mathrm{~V}$ communication have been developed to ensure interoperability in information exchange among vehicles e.g., ad-hoc communications over the IEEE $802.11 \mathrm{p}$ standard [2]. However, there are several inherent limitations of the IEEE $802.11 \mathrm{p}$ technology due to the dynamic nature of the vehicular communications environment and the stringent quality of service (QoS) requirements, such as scalability issues, potentially unbounded channel access delay, difficulty to ensure QoS guarantees, etc. [3-6]. Moreover, due to its limited radio range and lack of pervasive roadside infrastructure, IEEE $802.11 \mathrm{p}$ networks can only provide intermittent and short connections between the vehicles and the infrastructure. To address these issues, 3GPP has already introduced a standardized system (LTE-V2X) that can operate in a wide geographical area and meet the requirements of $\mathrm{V} 2 \mathrm{X}$ [7]. LTE-V2X has been designed for specific use cases [8], taking into account services and parameters defined in the first release of ETSI ITS [2] such as safety applications, traffic efficiency, and infotainment services. In turn, 5G V2X, referred to as enhanced V2X (eV2X), will address more advanced use cases such as cooperative intersection control, lane merging, and platooning, which have more stringent requirements [9].

In LTE-V2X, the V2V communications can be performed using two transmission modes, namely cellular mode and sidelink (SL) mode. The sidelink mode refers to the direct V2V communication that allows a User Equipment (UE) at a vehicle to communicate and transmit data directly to other UEs over the PC5 interface. Instead, the cellular mode uses the Uu interface (i.e. the radio interface between the UE and the base station) with a two-hop transmission via a base station, i.e. involving an uplink (UL) and a downlink (DL) transmission. The integration of the sidelink with cellular networks is a key technology to efficiently support V2V applications and to meet their latency requirements. It is worth mentioning that, although the support for V2X sidelink communications was already standardized in 3GPP for LTE [7], V2X sidelink is not yet included in the current release 15 of $5 \mathrm{G}$ New Radio (NR) specifications, but it is subject to study for current release 16 [10].

Based on the above considerations, this paper proposes a novel mode selection and resource reuse strategy to select the appropriate mode of operation and decide the amount of resource blocks (RBs) for $\mathrm{V} 2 \mathrm{~V}$ links in cellular and sidelink modes, where multiple $\mathrm{V} 2 \mathrm{~V}$ links may share the same radio resources. Such a strategy would be useful in order to face different challenges in terms of capacity, latency, reliability, and scalability caused by the increase in network size and demands for radio resources.

The rest of this paper is organized as follows. In Section II we present the relevant related works and summarize the contributions of the paper. Section III introduces the system model and assumptions. Section IV discusses the proposed solution. Section V presents the performance evaluation followed by the conclusions in Section VI.

\section{RELATED WORKS}

The widely deployed cellular network, assisted with device-to-device (D2D) communications, can provide a 
promising solution to support $\mathrm{V} 2 \mathrm{~V}$ communications. There have been different considerable works that have been presented to explore mode selection and resource optimization in direct D2D communications taking into account the interference situation [11-13] when sharing the radio resources and the quality of the links for both cellular and D2D links [1420]. In [11], joint mode selection, channel assignment, and power control in D2D communications are investigated. They proposed low-complexity algorithms aiming at maximizing the overall system throughput while guaranteeing the signal-to noise-and-interference ratio of both D2D and cellular links according to different network loads. A holistic approach for D2D mode selection and interference alignment technique for interference management is proposed in [12]. Reference [13] proposed a joint D2D mode selection and resource allocation scheme in order to maximize the system sum rate while meeting the successive interference cancellation (SIC) decoding constraint. New mode selection schemes for D2D-enabled cellular communications are proposed and evaluated in [14-16]. The proposed scheme is based on D2D distance between given users to trigger the D2D mode. Similarly, [17] proposed a mode selection strategy to determine the mode of operation and allow D2D pairs to flexibly reuse the radio resources of cellular users to improve the quality of D2D links. The authors in [18] studied the mode selection problems in a multi-mode and multi-pair D2D network, where the eNB can assign one of the three D2D communication modes including local route mode, direct D2D mode, and relay D2D mode. An online learning technique that leverages combinatorial multi-armed bandits (CMAB) is proposed in [19] to tackle the combinatorial nature of the mode selection and resource allocation (MS\&RA). In turn, [20] proposed a dynamic mode selection and subchannel allocation for an orthogonal frequency-division multiple access (OFDMA) cellular network with D2D communications to minimize the average end-to-end delay performance under the dropping probability constraint.

Although the above works have proposed different approaches for mode selection, none of them has considered jointly the mode selection between sidelink and cellular modes taking into account the quality of the links between V2V users in sidelink mode and between the base station and the vehicles in cellular mode, and the available resources. In this respect, in our previous work [21], we proposed a novel algorithm for mode selection that takes into account the quality of these links and the network traffic load situation.

Based on all the above, the main contribution of this paper is the proposal and analysis of a novel mode selection to decide when it is appropriate to use one or the other mode for the involved vehicles and the amount of radio resources for each mode. The proposed mode selection strategy takes into account the quality of the links between V2V users in sidelink mode and between the base station and the vehicles in cellular mode, and the available resources. Different from [21], the proposed mode selection decides the amount of resources to be allocated to $\mathrm{V} 2 \mathrm{~V}$ links in each mode and allows reusing RBs between different SL users, provided that the interference constraints are met. Our proposed mode selection strategy targets to achieve further improvements in the network performance (i.e., in terms of resource utilization, V2X service latency, achievable data rate, and outage probability).

\section{SYSTEM MODEL}

The considered scenario assumes a cellular Next Generation Radio Access Network (NG-RAN) with different gNodeBs (gNBs) deployed along a highway [22]. A roadside unit (RSU) supporting V2X communications is attached to each gNB. A flow of several independent vehicles move along a straight highway, as illustrated in Fig. 1. The highway segment is divided into sub-segments (clusters) by sectioning the road into smaller zones according to the length of the road. It is assumed that each vehicle includes a User Equipment (UE) that enables communication with the UEs in the rest of vehicles in the same cluster. Clusters are numbered as $j=1, \ldots, C$, and the vehicles in the $j$-th cluster are numbered as $i=1, \ldots, V(j)$. The vehicles in the highway are assumed to enter the cell coverage following a Poisson process with arrival rate $\lambda_{\mathrm{v}}$. The association between clusters and vehicles is managed and maintained by the RSU based on different metrics (e.g., position, direction, speed, and link quality) through periodic exchange of status information.

This paper assumes that $\mathrm{V} 2 \mathrm{~V}$ communication between vehicles can be performed either in cellular or in sidelink mode. In cellular mode, each UE communicates with each other through the $\mathrm{Uu}$ interface in a two-hops transmission (i.e. uplink and downlink) via the gNB while in sidelink mode, direct V2V communications are established over the PC5 interface. We assume that, when sidelink transmissions are utilized, every vehicle can multicast the $\mathrm{V} 2 \mathrm{~V}$ messages directly to multiple vehicles of the same cluster $1 \leq i \leq \mathrm{V}(j)$ using one-to-many technology.

The number of available RBs in UL and DL are denoted as $\mathrm{N}_{R B}^{U L}, \mathrm{~N}_{R B}^{D L}$, respectively. Regarding the radio resources for $\mathrm{V} 2 \mathrm{~V}$ in uplink and sidelink, we assume that, like in LTE, the sidelink RBs are a subset of the uplink RBs and all clusters in SL get the resource blocks from the UL resource blocks.

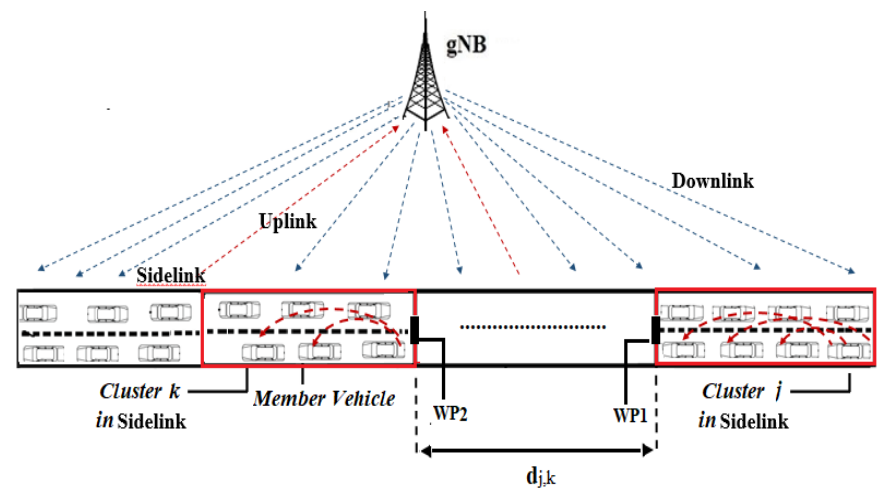

Fig. 1. The system model of the cellular network with sidelink V2V.

\section{PROPOSED SOLUTION}

This paper considers a mode selection and RBs reuse strategy (MS-RBRS), in which the system keeps track of the performance of both modes, i.e. SL and cellular, for each cluster in terms of the received signal and the availability of resources. The proposed algorithm operates periodically in time windows of duration $T$ and is detailed in the pseudo-code of Algorithm 1. We introduce an indicator $\alpha_{j}$ to reflect the operation mode of 
the $j$-th cluster so that $\alpha_{j}=1$ if vehicles within the cluster operate in sidelink mode and $\alpha_{j}=0$ if they operate in cellular mode. The proposed strategy assumes that cluster $j$ is working in mode $\alpha_{j}$ at a given time window, and the algorithm determines the mode for the next time window. To make this decision, the proposed mode selection strategy considers the following conditions for cluster $j$ :

1- It must ensure high signal quality by connecting V2V links to the mode with better signal-to-interference plus-noise ratios (SINRs). Let $\gamma_{j, i}^{U L}(\mathrm{t})$ and $\gamma_{j, i}^{D L}(\mathrm{t})$ denote the measured SINR for the $i$-th vehicle in the uplink and downlink of cellular mode, respectively, and $\gamma_{j, i, i^{\prime}}^{S L}$ the SINR in the sidelink between the $i$-th and the $i$-th vehicles of the $j$-th cluster. Each vehicle transmits the information about the received SINR from the gNB and other transmitting vehicles respectively to the gNB. Then, the average SINR for each cluster of vehicles is computed for each mode of operation and compared to each other (line 4).

The average SINR for all the UEs in uplink and downlink transmissions within each cluster in a time window $T$ can be statistically estimated as follows:

$$
\bar{\gamma}_{j}^{x}=\frac{1}{T \cdot V(j)} \sum_{t=1}^{T} \sum_{i=1}^{V(j)} \gamma_{j, i}^{x}(t)
$$

where $x \in\{\mathrm{UL}, \mathrm{DL}\}$. As for the sidelink mode, since multicast technology is utilized to transmit the packets from vehicle $i$ to other vehicles within the cluster for $1 \leq i \leq \mathrm{V}(\mathrm{j}), i^{\prime} \neq i$, the average of SINR for all the UEs within cluster $j$ in a time window $T$ is calculated as:

$$
\bar{\gamma}_{j}^{S L}=\frac{1}{T . V(j) .(V(j)-1)} \sum_{t=1}^{T} \sum_{i=1}^{V(j)} \sum_{\substack{i^{\prime}=1 \\ i^{\prime} \neq i}}^{V(j)} \gamma_{j, i, i^{\prime}}^{S L}(t)
$$

2-The V2V links to be established in sidelink or cellular mode must ensure that the amount of required RBs by cluster $j$ is less than the number of RBs available for the mode in which the vehicles are to be switched and operated. i.e., for every $j=1, \ldots, C$, the following condition is checked (lines 6, 10):

$$
\Gamma_{\mathrm{j}}^{x} \leq \mathrm{N}_{R B}^{x}-\rho^{x}
$$

where $\mathrm{N}_{R B}^{x}$ is total the number of RBs in the link $x \in\{\mathrm{UL}, \mathrm{DL}\}$. $\rho^{x}$ is the total number of RBs that have been already allocated to link $x \in\{\mathrm{UL}, \mathrm{DL}, \mathrm{SL}\}$ by the algorithm 1 (lines $2,8,9,12) . \Gamma_{\mathrm{j}}^{x}$ is the average number of required RBs from V2X users in cluster $j$ in link $x \in\{\mathrm{UL}, \mathrm{DL}, \mathrm{SL}\}$ over the time window $T$, given by:

$$
\Gamma_{\mathrm{j}}^{x}=\frac{\sum_{t=0}^{T} \sum_{i=1}^{V(j)} m(j, i, t) \cdot S_{m}}{T \cdot S P_{\text {eff }, x} \cdot B \cdot T_{s}}
$$

where $m(j, i, t)$ is the number of transmitted packets by the vehicles of the $j$-th cluster at each time $t$ within the time window
$T, S P_{\text {eff, } x}$ is the spectral efficiency associated to the modulation and coding scheme to be used in the $x$ link, $T_{s}$ is the TTI duration and $B$ the bandwidth per RB.

Algorithm 1: Mode selection and RBs reuse scheme 1. Inputs:

$\mathrm{N}_{R B}^{x}:$ Number of RBs in the link $x \in\{\mathrm{UL}, \mathrm{DL}\}$.

$C$ : number of clusters.

$V(j)$ : vehicles in cluster $j$

$\gamma_{j, i}^{U L} \gamma_{j, i}^{D L} \gamma_{j, i, i^{\prime}}^{S L}$

2. Initialization: $\rho^{x}=0$

3. For each cluster $j=1, \ldots, C$

4. Compute the average value of $\bar{\gamma}_{j}^{U L} \quad \bar{\gamma}_{j}^{D L} \quad \bar{\gamma}_{j}^{S L}$ among all vehicles within cluster $j$ using eqs. (1) and (2);

5. If $\min \left(\bar{\gamma}_{j}^{U L}, \bar{\gamma}_{j}^{D L}\right)>\bar{\gamma}_{j}^{S L}$

6.

If $\Gamma_{\mathrm{j}}^{x} \leq \mathrm{N}_{R B}^{x}-\rho^{x}$ \{ check that the required RBs by cluster $\mathrm{j}$ are available in each link $x \in$ [UL, DL]\}.
7.
7. $\alpha_{j}=0 \quad\{$ cluster $j$ operates in cellular mode
9. $\quad \rho^{D L}=\rho^{D L}+\Gamma_{\mathrm{j}}^{D L}$

10. Else If $\Gamma_{\mathrm{j}}^{S L} \leq \mathrm{N}_{R B}^{U L}-\rho^{U L}$ \{ check that the required RBs by cluster $\mathrm{j}$ are available in $\mathrm{UL}$ $\alpha_{j}=1 \quad$ cluster $j$ operates in sidelink mode\}

$$
\rho^{S L}=\rho^{S L}+\Gamma_{\mathrm{j}}^{S L}
$$

Else

\{Check for RBs reuse in SL\}

$\alpha_{j}=1$ \{ cluster $j$ operates in SL mode\}

Find the cluster $k$ with $\max d_{j, k}$

compute $\gamma_{j, \mathrm{k}}$ between the worst-case

positions in clusters $k$ and $j$

17. If $\gamma_{j, k} \geq \gamma_{T H}$

18. RBs can be reused in both clusters in SL

19. Else

20. RBs can not be reused in both clusters in SL

21.

22. End

23. Else

24.

25. End

26.End

27.Output: $\alpha_{j} \quad j=1, . ., C$, Number of RBs for $\mathrm{UL}=\rho^{U L}$, Number of RBs for $\mathrm{SL}=\rho^{S L}$,

3- In order for the V2V links within cluster $j$ in SL mode to reuse other radio resources used by other V2V links in cluster $k=1, \ldots, C, k \neq j$ operating in SL mode (cluster $k$ with $\alpha_{k}=1$ ), it must be ensured that the SINR between cluster $j$ and cluster $k$ 
is higher than a threshold ( i.e., $\gamma_{j, k} \geq \gamma_{T H}$ ). Since the positions of the transmitters and receivers will change dynamically, in order to compute the SINR between cluster $j$ and cluster $k$ on a long-term basis that is valid for any condition, let us assume the worst case situation between any transmitter of cluster $j$ and any receiver of cluster $k$ (i.e., the positions of the vehicles that lead to lowest SINR). The worst case positions are denoted as WP1 and WP2 and shown in Fig. 1. Based on this, the SINR between cluster $j$ and cluster $k, \gamma_{j, k}$ is calculated as follows:

$$
\gamma_{j, \mathrm{k}}=\frac{P_{T} G_{T} G_{R}}{L_{j, k}\left(P_{n}+I_{j, k}\right)}
$$

where $P_{T}$ is the transmitted power in one RB, $G_{T}$ is the antenna gain of the transmitter, $G_{R}$ is the antenna gain of the receiver, $L_{j, k}$ is the path loss at distance $d_{j, k}$, and $P_{n}$ is the power of the additive white Gaussian noise (AWGN). $I_{j, k}$ is the interference power received by the interfered receiver of cluster $j$ from the interferer transmitter of cluster $k$ in the worst case positions WP1 and WP2.

Based on the above conditions, the mode selection criterion in Algorithm1 is as follows: if the average values of SINR for both uplink and downlink in the $j$-th cluster are higher than the average value of SINR for SL (i.e. $\left.\min \left(\bar{\gamma}_{j}^{U L}, \bar{\gamma}_{j}^{D L}\right)>\bar{\gamma}_{j}^{S L}\right)$, the algorithm will move on to check that there are sufficient physical resources to be used for serving the V2V links in cellular mode before taking the decision for switching (lines $5,6)$. If one of the conditions is not satisfied, the cluster will stay in sidelink mode $\left(\alpha_{j}=1\right)$ (lines 11,14$)$. Besides, when there are no more available RBs left for data transmissions, the algorithm allows users of cluster $j$ to reuse the resources of V2V links within cluster $k$ (i.e., where $k$ refers to the cluster with maximum distance $d_{j, k}$ from cluster $j$ ) if the minimum requirement of the $\operatorname{SINR}$ (i.e., $\gamma_{j, k} \geq \gamma_{t h}$ ) is met. Otherwise, the system will be in outage conditions.

\section{PERFORMANCE EVALUATION}

Our simulation model is based on two cells configured with two gNBs. Each gNB handles one cell with a channel organized in $80 \mathrm{RBs}$ for UL and $80 \mathrm{RBs}$ for DL. Each RB is composed of 12 subcarriers with subcarrier separation $\Delta f=30 \mathrm{kHz}$, which corresponds to one of the 5G NR numerologies defined in [23]. The model considers vehicular UEs communicating through cellular mode (uplink / downlink) and via sidelink (direct V2V). The users move along a 2-lane highway and are assumed to enter the cell coverage following a Poisson process with arrival rate $\left(\lambda_{\mathrm{v}}\right)$. All relevant system and simulation parameters are summarized in Table I. The presented evaluation results intend to assess and illustrate the performance of the proposed solution in terms of latency, packet success rate and RB utilization. As a reference for comparison, we assume a mode selection strategy denoted as "Mode Selection Signal- Based (MSSB)", which takes into account only the quality of the links and is inspired from the work in [21].
TABLE I. SIMULATION PARAMETERS

\begin{tabular}{|c|c|}
\hline Parameter & Values \\
\hline Cell radius & $500 \mathrm{~m}$ \\
\hline \multirow{2}{*}{ Number of RBs per cell } & $\mathrm{N}_{R B}^{U L}=80 \mathrm{RBs}$ \\
\hline & $\mathrm{N}_{R B}^{D L}=80 \mathrm{RBs}$ \\
\hline Frequency & $5.9 \mathrm{GHz}$ \\
\hline$\Delta f$ & $30 \mathrm{kHz}$ \\
\hline Path loss model & $\begin{array}{l}\text { The path loss and the LOS } \\
\text { probability for cellular mode are } \\
\text { modeled as in [24]. In sidelink mode, } \\
\text { all V2V links are modeled based on } \\
\text { freeway case (WINNER+B1) with } \\
\text { hexagonal layout [ITU-R] [25]. }\end{array}$ \\
\hline Height of the gNB & $10 \mathrm{~m}$ \\
\hline $\begin{array}{c}\text { Base station receiver } \\
\text { noise figure }\end{array}$ & $9 \mathrm{~dB}$ \\
\hline $\begin{array}{l}\text { Spectral efficiency } \\
\text { model to map SINR. }\end{array}$ & $\begin{array}{l}\text { Model in section A.1 of [26]. The } \\
\text { maximum spectral efficiency is } 1 \\
\text { b/s/Hz. }\end{array}$ \\
\hline $\begin{array}{c}\text { Shadowing standard } \\
\text { deviation }\end{array}$ & $3 \mathrm{~dB}$ in LOS and $4 \mathrm{~dB}$ in NLOS. \\
\hline $\begin{array}{c}\text { Base station antenna } \\
\text { gain }\end{array}$ & $5 \mathrm{~dB}$ \\
\hline Length of the highway & $3 \mathrm{Km}$ \\
\hline Number of lanes & 2 in one direction \\
\hline Lane width & $4 \mathrm{~m}$ \\
\hline Size of clusters & 12 per cell \\
\hline Size of cluster & 250 \\
\hline Vehicular UE height & $1.5 \mathrm{~m}$ \\
\hline $\begin{array}{c}\text { Transmitted power per } \\
\text { RB } \\
\end{array}$ & $23 \mathrm{dBm}$ \\
\hline UE antenna gain & $3 \mathrm{~dB}$ \\
\hline Vehicle speed & $80 \mathrm{Km} / \mathrm{h}$ \\
\hline UE Noise power $P_{n}$ & $-114 \mathrm{dBm}$ \\
\hline Vehicle arrival rate $\lambda_{\mathrm{v}}$ & Varied from 1 to 8 vehicles/s \\
\hline Packet arrival rate $\lambda_{\mathrm{a}}$ & 1 packets/s \\
\hline Message size $\left(\mathrm{S}_{\mathrm{m}}\right)$ & $\{400,800,1200\}$ bytes \\
\hline TTI duration $\left(\mathrm{T}_{\mathrm{s}}\right)$ & $1 \mathrm{~ms}$ \\
\hline Time window $\mathrm{T}$ & $3 \mathrm{~s}$ \\
\hline$\gamma_{\mathrm{TH}}$ & $14 \mathrm{~dB}$ \\
\hline
\end{tabular}

Fig. 2 depicts the throughput delivered in Kbits/sec for V2X service aggregated for sidelink and uplink in each cell. The figure illustrates the behavior of the proposed solution and the MSSB reference scheme. Results are presented for different vehicle arrival rates and message sizes. Here, we can observe that the proposed solution outperforms the MSSB reference scheme in terms of throughput. The proposed scheme achieved throughput of $451 \mathrm{~kb} / \mathrm{s}$ in uplink when the vehicle arrival rate $\left(\lambda_{\mathrm{v}}\right)$ is 8 vehicles/s and the message size is 1200 bytes. For the MSSB reference scheme, the throughput is only $402 \mathrm{~Kb} / \mathrm{s}$ in uplink (i.e. proposed approach achieves a relative gain of $12 \%$ ). The reason comes from the fact that, when the vehicle arrival rate $\lambda_{\mathrm{v}}$ of V2X UEs is increased, more users will use the network and this will 
increase the number of $\mathrm{V} 2 \mathrm{X}$ packets and request more $\mathrm{RBs}$ to be used in transmissions. Then, in this situation, the proposed solution ensures more RBs for transmitting data by taking advantage from the reuse of RBs for other SL V2V links that meet the interference requirements, while the MSSB reference scheme does not exploit this reuse.

In Fig.3, we investigate the probability of having outage at a certain point in time (i.e. the probability that there are no sufficient RBs to serve all the transmission requests). The outage probability of the proposed solution and reference scheme are plotted against the V2X vehicle arrival rate $\lambda_{\mathrm{v}}$. We can clearly observe that, increasing the traffic load, leads to an increase in the outage probability. It can be also noted that our proposed strategy can substantially reduce the probability of having outage thanks to the resource reuse that guarantees the availability of more resources.

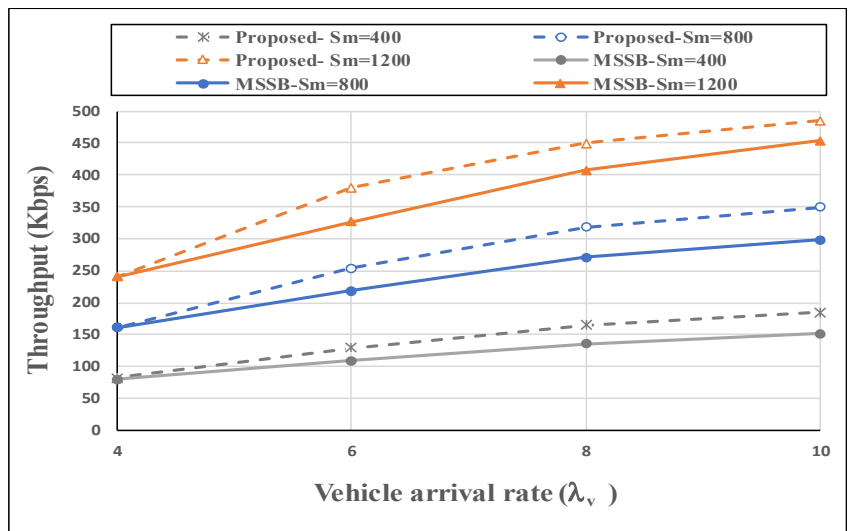

Fig.2. Aggregated UL and SL throughput as a function of the V2X UE arrival rate $\lambda_{v}$ (vehicles/s).

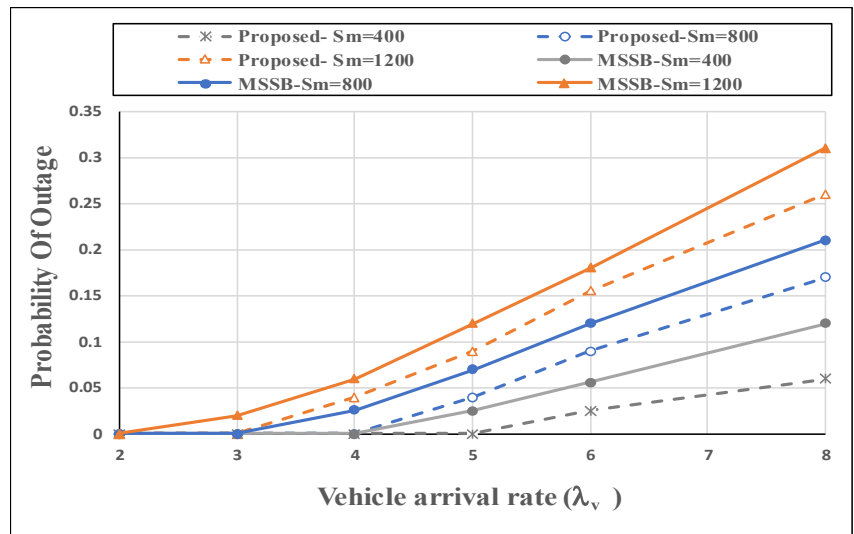

Fig. 3. Outage probability vs V2X UE arrival rate $\lambda_{\mathrm{v}}$ (vehicles/s).

Fig. 4 illustrates the average latency for V2X service caused by channel access delay and the transmission delay. Latency is measured as the time spent by a packet in the system, from the time it is generated until it has been transmitted. We can clearly observe from Fig. 4 that the average latency increases for all the approaches when vehicle arrival rate $\lambda_{\mathrm{V}}$ is increased, because more vehicles will use the network and request more RBs to be used for the transmissions. This causes an increase in the waiting time and therefore increases the latency. From the presented results, we notice that the proposed solution reduces the latency compared to MSSB reference scheme.
Fig. 5 presents the RB utilization for $\mathrm{V} 2 \mathrm{X}$ service in the UL in each cell as a function of the vehicle arrival rate $\left(\lambda_{\mathrm{V}}\right)$ for V2X users. The figure shows that there is a marked increase in RBs utilization for all the approaches when vehicle arrival rate is increased. The figure also shows that our proposed solution maintains the same RBs utilization as the MSSB reference scheme in different load scenarios (note that the results for the two schemes are overlapped in the figure). This is because the proposed approach does not allocate additional RBs to serve the traffic and improves the performance in terms of throughput, probability of outage, and latency, but reuses other radio resources used by other SL V2V links that meet the interference requirements.

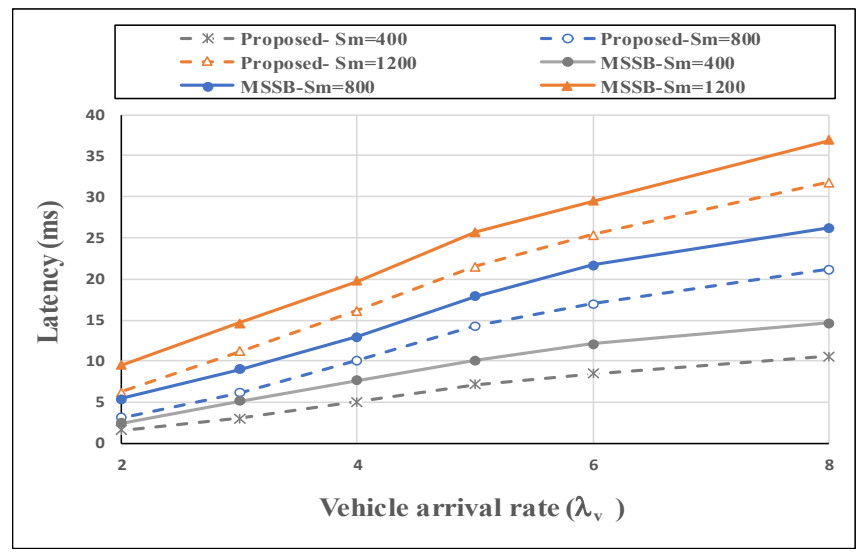

Fig. 4. Average Latency vs V2X UE arrival rate $\lambda_{v}$ (vehicles/s).

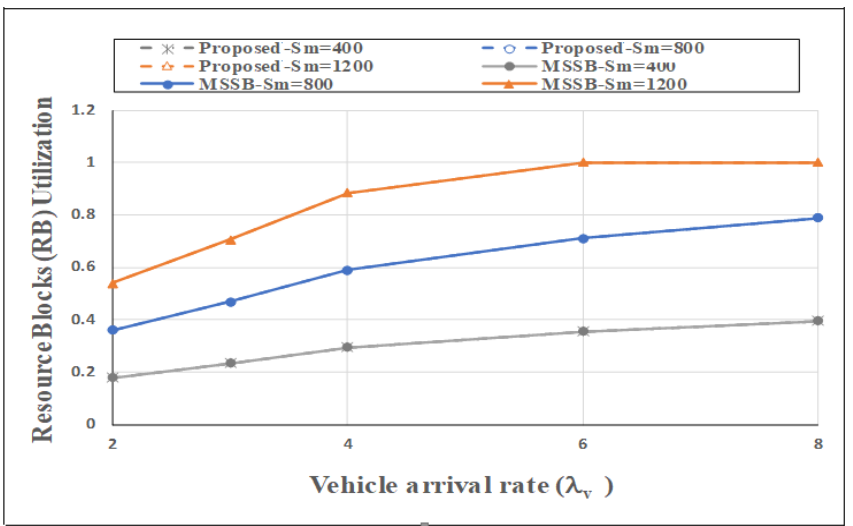

Fig. 5. Uplink RB utilization as a function of the V2X UE arrival rate $\lambda_{v}$ (vehicles/s)

\section{CONCLUSIONS}

In this paper, we have proposed a novel mode selection and resource reuse strategy for $\mathrm{V} 2 \mathrm{~V}$ communications to decide, on the one hand, when it is appropriate to select sidelink or cellular modes and, on the other hand, the amount of resources to be allocated to V2V links in each mode. The proposed approach takes into account the quality of the links between $\mathrm{V} 2 \mathrm{~V}$ users in sidelink mode and between the base station and the vehicles in cellular mode, as well as the available resources. In addition, the proposed approach allows $\mathrm{V} 2 \mathrm{~V}$ links in SL to reuse RBs that have been reassigned to $\mathrm{V} 2 \mathrm{~V}$ links within other clusters operating in SL if a minimum signal to noise and interference ratio requirement is met. 
Extensive simulations were conducted to validate and analyse the performance of our proposed solution. This strategy has been compared against a reference approach that considers only the quality of the links. Simulation results have shown the capability of the proposed algorithm to allocate the resources efficiently and improve the network performance in terms of throughput, outage probability and latency.

\section{ACKNOWLEDGMENT}

This work was supported in part by the Spanish Research Council and FEDER funds under SONAR 5G grant (ref. TEC2017-82651-R), and in part by the Iraqi University of Technology.

\section{REFERENCES}

[1] 5GCARS. "Final 5G V2X Radio Design" [Online]. May 2019. https://5gcar.eu/wp-content/uploads/2019/06/5GCAR_D3.3_v1.0.pdf.

[2] ETSI TC ITS, Intelligent Transport Systems (ITS); Vehicular Communications; Basic Set of Applications; Definitions, Std. ETSI TR 102638 V1.1.2, 2015.

[3] 5G Americas. "V2X Cellular Solutions" [Online]. October 2016. http://www.5gamericas.org/files/2914/7769/1296/5GA_V2X_Report_FI NAL for upload.pdf.

[4] G. Araniti, C. Campolo, M. Condoluci, A. Iera, and A. Molinaro, "LTE for vehicular networking: A survey," IEEE Commun. Mag., vol. 51, no. 5, pp. 148-157, May 2013.

[5] X. Cheng, L. Yang, and X. Shen, "D2D for intelligent transportation systems: A feasibility study," IEEE Trans. Intell. Transp. Syst., vol. 16, no. 4, pp. 1784-1793, Aug. 2015.

[6] C. Lottermann, M. Botsov, P. Fertl, and R. Mullner, "Performance evaluation of automotive off-board applications in LTE deployments," in Proc. IEEE Veh. Netw. Conf., Seoul, Korea, Nov. 2012, pp. 211-218.

[7] 3GPP TR 36.300, "Evolved Universal Terrestrial Radio Access (EUTRA) and Evolved Universal Terrestrial Radio Access Network (EUTRAN); Overall description; Stage 2 (v14.3.0, Release 15), June 2019.

[8] 3GPP TR 22.885, "Study on LTE support for Vehicle to Everything (V2X) services", v14.0.0, Dec. 2015.

[9] 3GPP TR 22.886, "Study on enhancement of 3GPP Support for 5G V2X Services", v15.1.0, Mar. 2017.

[10] 3GPP TR 38.885, "Study on NR Vehicle-to-Everything (Release 16)", v16.0.0, Mar. 2019.

[11] G. Yu, L. Xu, D. Feng, R. Yin, "Joint Mode Selection and Resource Allocation for Device-to-Device Communications", IEEE Trans. Commun., vol. 62, no. 11, pp. 3814_3824, Nov. 2014.

[12] H. Chou and R. Y. Chang, "Joint Mode Selection and Interference Management in Device-to-Device Communications Underlaid MIMO Cellular Networks", IEEE Trans. Commun., vol. 16, no. 2, pp. 11201134 , Feb. 2017.

[13] Y. Dai, M. Sheng, and X. Shen, "Joint Mode Selection and Resource Allocation for D2D-Enabled NOMA Cellular Networks", IEEE Trans. On Vehicular Technology, vol. 68, no. 7, pp. 6721-6733, Jul. 2019.

[14] S. Bulusu, B. Mehta, and S. Kalyanasundaram, "Rate Adaptation, Scheduling, and Mode Selection in D2D Systems with Partial Channel Knowledge", IEEE Trans. Commun., vol. 17, no. 2, pp. 1053_1065, Feb. 2018.

[15] A. Omri, and M. O. Hasna, "A Distance-Based Mode Selection Scheme for D2D-Enabled Networks with Mobility", IEEE Trans. Commun., vol. 17, no. 7, pp. 4326 4340, Jul. 2018.

[16] G. Wang, S. Zhou, Z. Niu, "Mode Selection in UAV-aided Vehicular Network: an Evolutionary Game Approach", in Proc. IEEE Wireless Commun. Conf., 2018.

[17] P. Zeng, X. Li, "A Strategy of Reusing Mode Selection for Device to Device Communication", 14th International Conference on Natural Computation, Fuzzy Systems and Knowledge Discovery (ICNC-FSKD), 2018. pp. 1231-1236.

[18] Ch. Chen, Chi. Sung, and H.Chen , "Capacity Maximization Based on Optimal Mode Selection in Multi-Mode and Multi-Pair D2D Communications Networks with Mobility", IEEE Trans. On Vehicular Technology, vol. 68, no. 7, pp. 5624_5634, Jul. 2019.
[19] A. Ortiz, A. Asadi, and M. Engelhardt, "CBMoS: Combinatorial Bandit Learning for Mode Selection and Resource Allocation in D2D Systems", IEEE jour. On selected areas in communications, vol. 37, no. 10, pp. 2225-2238, Oct. 2019.

[20] Lei Qingyun Hao,Zhangdui Zhong, "Mode Selection and Resource Allocation in Device-to-Device Communications With User Arrivals and Departures". IEEE Access : 5209-5222 (2016).

[21] H. D. R. Albonda and J. Pérez-Romero, "An efficient mode selection for improving resource utilization in sidelink V2X cellular networks", in Proc. IEEE 23rd Int.Workshop Comput. Aided Modeling Design Commun. Links Netw. (CAMAD), Barcelona, Spain, Sep. 2018, pp. 1_6.

[22] 3GPP TS38.401, "NG-RAN; Architecture Description (Release 15)", v15.2.0, Jun. 2018.

[23] 3GPP TS 38.211, "NR; Physical Channels and Modulation (Release 15)", v15.2.0, Jun. 2018

[24] Report ITU-R M.2135 "Guidelines for evaluation of radio interface technologies for IMT-Advanced”, 2009.

[25] WINNER II "Channel Models", D1.1.2 V1.2., available at http://www.cept.org/files/1050/documents/winner $\% 20 \% 20$ final $\% 20 \mathrm{r}$ report.pdf.

[26] 3GPP TR 36.942 v15.0.0, "Radio Frequency (RF) system scenarios", September, 2018. 